\title{
Electronically Coupled Gold Nanoclusters Render Deep-Red Emission with High Quantum Yields
}

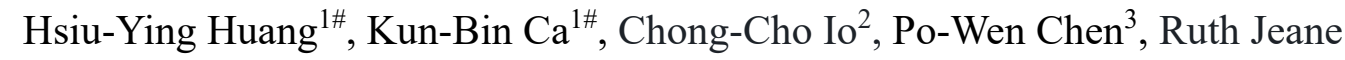
Soebroto $^{2}$, Ji-Lin Shen ${ }^{1}$, Jui-Ming Yeh $^{4}$ and Chi-Tsu Yuan ${ }^{1,2 *}$

${ }^{1}$ Department of Physics, Chung Yuan Christian University, Taoyuan, Taiwan

${ }^{2}$ Master Program in Nanotechnology, Chung Yuan Christian University, Taoyuan, Taiwan

${ }^{3}$ Physics Division, Institute of Nuclear Energy Research, Taoyuan, Taiwan

${ }^{4}$ Department of Chemistry, Chung Yuan Christian University, Taoyuan, Taiwan

\section{CORRESPONDING AUTHORS}

*E-mail: ctyuan@cycu.edu.tw (Chi-Tsu Yuan)

\section{Experimental section.}

\section{Materials.}

Gold(III) chloride trihydrate $\left(\mathrm{HAuCl}_{4} \cdot 3 \mathrm{H}_{2} \mathrm{O}\right)$, L-Glutathione in the reduced form $(\mathrm{GSH})$ and(3-Aminopropyl)trimethoxysilane (APTMS) were purchased from the SigmaAldrich. All of the reagents are of analytical grade and used without further treatment.

\section{Synthesis of Si-AuNCs.}

GSH-stabilized AuNCs were prepared as follows. Freshly prepared aqueous solution of GSH $(10 \mathrm{~mL}, 75 \mathrm{mM})$ was mixed with $\mathrm{HAuCl}_{4}(10 \mathrm{~mL}, 50 \mathrm{mM})$ under vigorous stirring at ambient temperature, for $5 \mathrm{~min}$. Until the mixture turned colorless, the solution was transferred into the microwave vessels and sealed. The reaction temperature was set at $90{ }^{\circ} \mathrm{C}$, which was reached in $3 \mathrm{~min}$ from room temperature, and the irradiation time 
was set for $3 \mathrm{~h}$. Then, the obtained product was stored in a refrigerator at $4{ }^{\circ} \mathrm{C}$ for further use. The as-prepared thiolate-stabilized AuNCs solution (1.4 ml) was concentrated by removing the solvents in a rotary evaporator at $50{ }^{\circ} \mathrm{C}$ and further dissolved in DI water $(0.8 \mathrm{ml})$ then mixed with APTMS $(4.8 \mathrm{ml})$. After sufficient stirring, a viscous and yellow-orange aminosilane-grafted AuNCs solution was obtained. Solid SiAuNC@silica composite was simply fabricated on glass substrates using sol-gel reaction. The thiolated AuNCs were mixed with aminosilane agents to form silanegrafted AuNCs (Si-AuNCs). The glass substrates were ultrasonically cleaned with acetone, deionized water and ethanol for $15 \mathrm{~min}$, respectively. After being treated by UV-ozone for $20 \mathrm{~min}$, these substrates can be readily used. The Si-AuNCs were dropped onto the glass substrate and can be further processed to form the thin films by doctorblading method (PFA-2010-S motorized film applicator, Proyes) at a speed of $10 \mathrm{~mm} / \mathrm{s}$ and then dried at $25^{\circ} \mathrm{C}$ for $2 \mathrm{~h}$. Finally, this sample was heated under an air flow oven at $50{ }^{\circ} \mathrm{C}$ for 2 weeks to fabricate solid Si-AuNC@silica composite.

\section{Characterization.}

The image of HR-TEM is taken using Field Emission Transmission Electron Microscope (FE-TEM, JEOL JEM-2100F). Fourier transform infrared (FTIR) spectra were collected at room temperature (on a Jasco FTIR-4100 spectrometer) from the sample prepared as pellets with $\mathrm{KBr}$ host. The surface areas and pore size distributions were measured using a Micromeritics ASAP 2020 M. The UV-Vis absorption spectra were recorded with V-750 UV-Vis spectrophotometer (Jasco). Steady-state and timeresolved PL measurements were performed based a spectrophotometers (Fluotime 300, PicoQuant). A xenon lamp were used as the excitation sources and the PL emission is collected by the PMT detector calibrated by the specific wavelength-response function 
of our system. The absolute QY measurement is carried out based on the same spectrophotometers integrated with an integrating sphere. TRPL were performed using a spectrometer integrated with a pulsed Xenon lamp as excitation sources based on time-correlated single-photon counting or multichannel scaling. Photomultiplier tubes with spectral ranges from $\sim 200$ to $\sim 900 \mathrm{~nm}$ was used as the detector. The measured PL was calibrated by our system response function.

Figure S1. Magnified absorbance spectra for both composites, showing newly emerged hump for the R-composite.

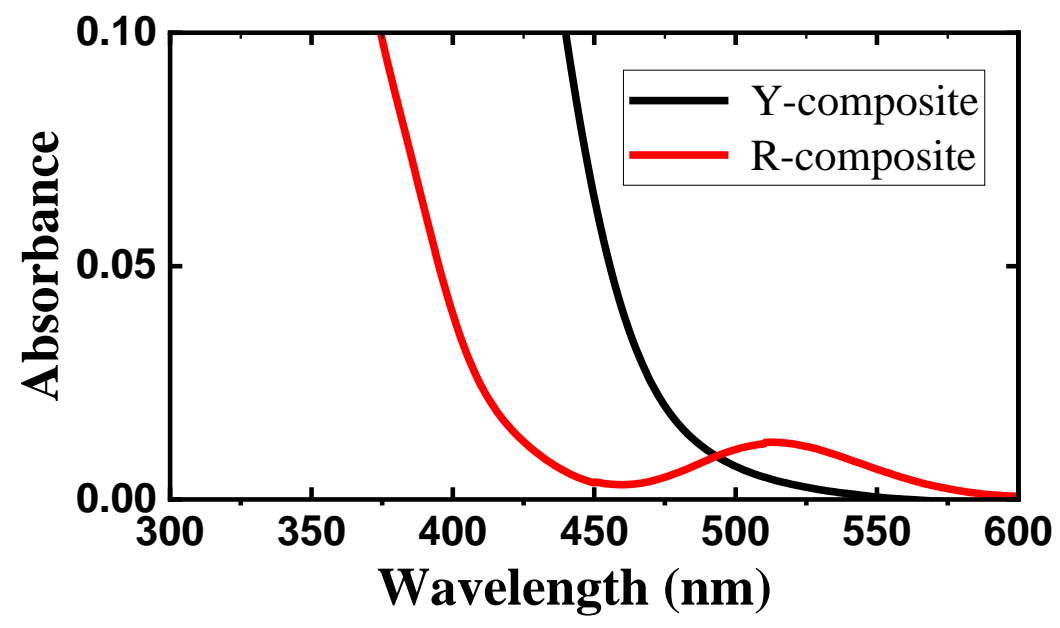


Figure S2. Time-resolved PL decay traces with fitting curves and fitting results.

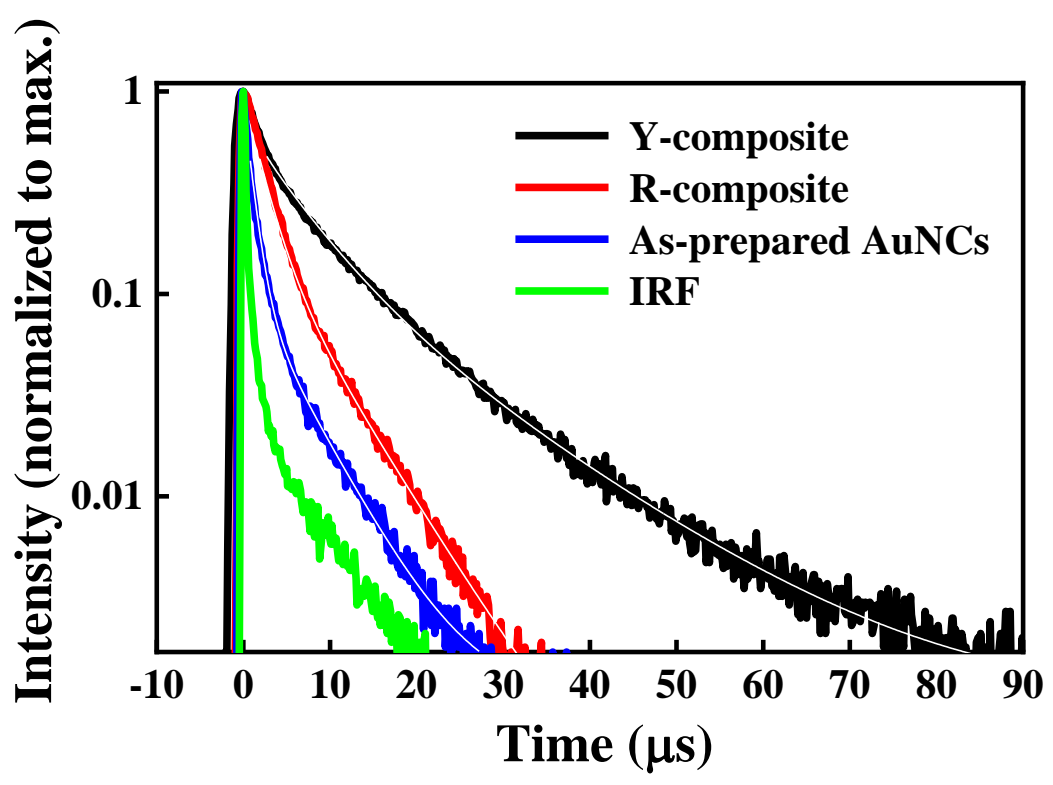

\begin{tabular}{|c|c|c|c|}
\hline & Y-composite & R-composite & $\begin{array}{c}\text { As-prepared } \\
\text { AuNCs }\end{array}$ \\
\hline $\mathrm{A}_{1}$ & 0.2 & 0.78 & 0.8 \\
\hline $\boldsymbol{\tau}_{\mathbf{1}}$ & $1 \mu \mathrm{s}$ & $2 \mu \mathrm{s}$ & $1 \mu \mathrm{s}$ \\
\hline $\mathrm{A}_{\mathbf{2}}$ & 0.5 & 0.22 & 0.11 \\
\hline $\boldsymbol{\tau}_{\mathbf{2}}$ & $5.8 \mu \mathrm{s}$ & $6.3 \mu \mathrm{s}$ & $5.4 \mu \mathrm{s}$ \\
\hline $\mathrm{A}_{3}$ & 0.18 & & \\
\hline $\boldsymbol{\tau}_{3}$ & $15 \mu \mathrm{s}$ & & \\
\hline$<\boldsymbol{\tau}>$ & $9.9 \mu \mathrm{s}$ & $4.0 \mu \mathrm{s}$ & $2.9 \mu \mathrm{s}$ \\
\hline
\end{tabular}


Figure S3. Calculation of the radiative and nonradiative decay rates.

\begin{tabular}{|l|c|c|c|}
\hline & Y-composite & R-composite & As-prepared AuNCs \\
\hline Radiative rates $\left(\mathrm{s}^{-1}\right)$ & $1.5 \times 10^{4}$ & $1.7 \times 10^{5}$ & $1.0 \times 10^{4}$ \\
\hline Nonradiative rates $\left(\mathrm{s}^{-1}\right)$ & $8.6 \times 10^{4}$ & $8.5 \times 10^{4}$ & $3.3 \times 10^{5}$ \\
\hline
\end{tabular}

Figure S4. High-resolution TEM image for Si-AuNC@silica composite prepared under acidic condition.

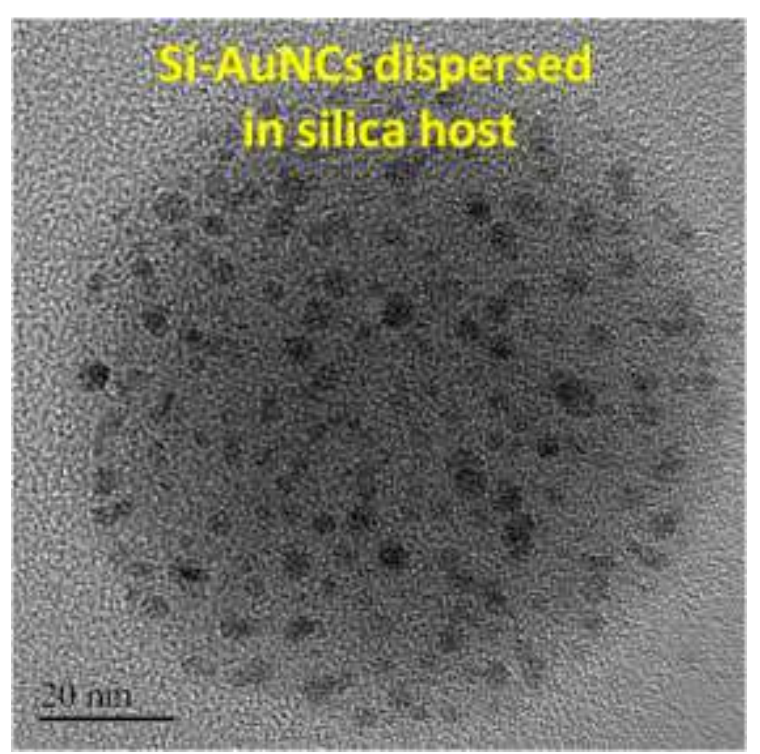

